\title{
On Adapting to Remote Learning for Healthcare Professionals
}

Barb Himes, IBCLC

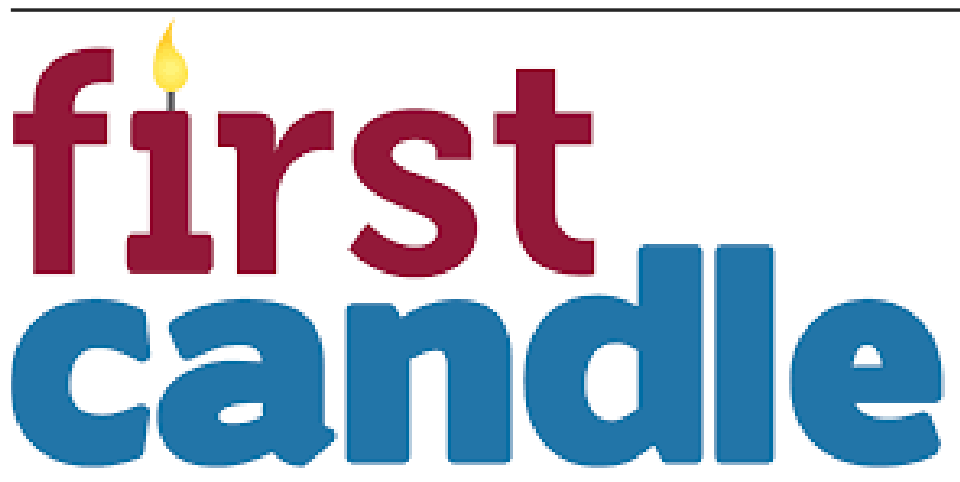

Saving babies. Supporting families.

First Candle's efforts to support families during their most difficult times and provide new answers to help other families avoid the tragedy of the loss of their baby are without parallel.

\section{"The COVID-19 pandemic has changed} and continues to change our health outlook, risk behaviors, and daily activities. For clinicians and health education professionals, it has forced us to be open to new ways of working with each other and with those we serve."

The COVID-19 pandemic has changed and continues to change our health outlook, risk behaviors, and daily activities. For clinicians and health education professionals, it has forced us to be open to new ways of working with each other and with those we serve.

Some of these options we have had to explore have resulted in new perspectives and results we might not have realized possible before, and which can leave us with more choices than we might have thought.

A case in point for us at First Candle has been conducting our Straight Talk for Infant Safe Sleep program during the past year and into this one, which has given us some lessons learned that might be useful to other health care providers.

Our mission is to reduce the rate of infant mortality due to Sudden Unexplained Infant Death (SUID), which includes Sudden Infant Death Syndrome (SIDS) and Accidental Strangulation and Suffocation in Bed (ASSB). This can be achieved by following the American Academy of Pediatrics (AAP) set of recommendations for infant safe sleep. These guidelines, updated every five years, have been in use since the original Back to Sleep campaign, developed by a coalition including NICHD, the AAP Task Force, NHLBI, HRSA, and the SIDS Alliance (now First Candle) in 1994.

In 2017 we strengthened our educational outreach with the launch of Straight Talk for Infant Safe Sleep, a train-the-professional education workshop designed for health care providers, including nurses, doulas, social service agencies, faith-based workers, and childcare providers.

The program, under which nurses can earn CEU contact hours, follows a collaborative approach designed not only to cover the AAP guidelines but to help frame them in the context of a given family's environment, recognizing many factors that influence a parent's safe sleep decisions. These can include family and cultural norms, socio-economic factors, past experience, and the desire to bond or breastfeed (which we recommend). The program also addresses unrecognized implicit bias from healthcare providers, which through qualitative feedback to First Candle and other organizations indicated a barrier to adopting the safe sleep guidelines. The normal training format is a five-hour session of instruction, general discussion, and breakout groups.

In 2019 we were scheduled to conduct five sessions hosted by a state's hospital association with its member hospitals' staff. Then the pandemic and lockdown happened. However, working with the association, we developed a combination of in-person and online schedules that enabled us to proceed. The in-person sessions were held in training rooms or the association's classrooms and followed COVID-19 protocols regarding sanitation, distancing, and personal protective equipment.

We realized that we were naturally moving into new territory for the sessions that would have to be online and so observed these not only for their stated purpose but also to assess how the format itself performed as a training method.

We learned several things, key among them that we could accom-

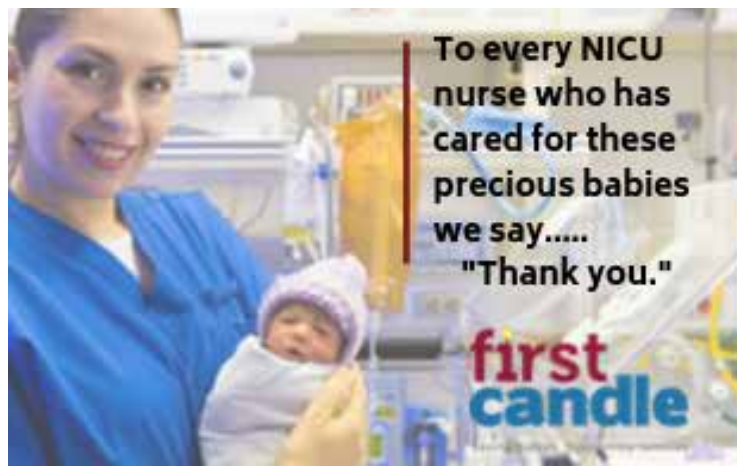

\section{Did you know that premature and low birth weight babies have a $4 \times$ greater risk for SIDS?}

At First Candle we're educating parents, grandparents and caregivers about safer sleep to make sure all babies reach their first birthday. Learn more at firstcandle.org 
modate more participants than in person. They were more forthcoming in responses having to do with their current understanding of infant safe sleep and their perceptions about human behaviors. We attribute this in part to the association's online format; their responses were not individually identified to the group but were read aloud and shared by the trainer.

In addition, because their responses came in simultaneously, there was no opportunity for first-out responses to influence subsequent ones. In the instances where several respondents did provide the same answer, we could share it once as an acknowledged collective response, saving everyone time.

A strong component of the Straight Talk program focuses on implicit bias, including recognizing it and how to address it when working with parents and families. Some thought that this part of the course's dynamics might be affected by the remote format, but we found it was not. Participants were able to engage in constructive individual self-revelation and group discussion.

\section{"A strong component of the Straight Talk} program focuses on implicit bias, including recognizing it and how to address it when working with parents and families. Some thought that this part of the course's dynamics might be affected by the remote format, but we found it was not. "

Post-course evaluations showed that $100 \%$ of the participants reported a heightened awareness of implicit bias, and $87 \%$ reported having implemented changes in the time period subsequent to taking the course, including sharing learnings with staff, increasing their patient prenatal education in hospital, and a more sensitive approach to working with families.

However, under the hospital association's format, we did not have a breakout session option, which we are working to include as we move forward with the online version.

And we are moving forward with it during this year and will continue to do so even as things gradually begin to open up again, making it a permanent option to our Straight Talk offerings. We expect that we may gain further understanding of how people learn via online platforms (e.g., some may find they are more forthcoming in writing vs. orally, and there may be different results among sessions depending upon levels of identification, and so on.)

\section{"Adult professional education is a different arena than online primary and secondary schooling, which involves a younger isolated population and has its own set of challenges. We look forward to learning more about this format's dynamics even as we pass learning on to our constituents."}

Adult professional education is a different arena than online primary and secondary schooling, which involves a younger isolated population and has its own set of challenges. We look forward to learning more about this format's dynamics even as we pass learning on to our constituents.

Disclosure: The author is the Executive Director and Chief Executive Officer of First Candle, Inc., a Connecticut not for profit 501c3 corporation.

NT
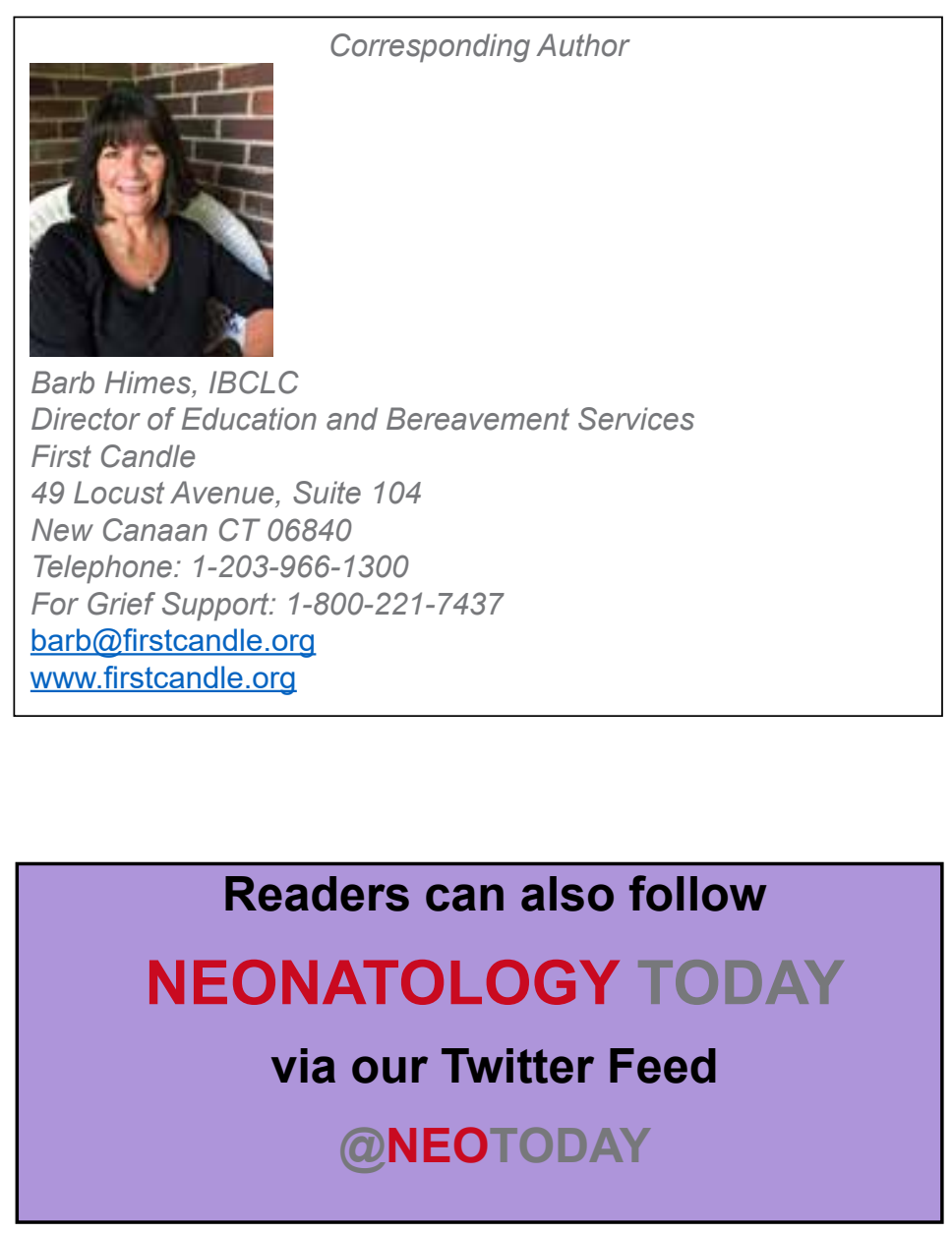